\title{
O INVESTIGADOR, O PROFESSOR UNIVERSITÁRIO E O DIRECTOR DO CENTRO DE ESTUDOS GEOGRÁFICOS*
}

SUZANNE DAVEAU ${ }^{1}$

Orlando Ribeiro é sobretudo conhecido como autor de obras geográficas, que tratam tanto de Portugal como do mundo lusófono, e que conheceram difusão muito desigual. Há páginas suas que figuram em manuais escolares ou em edições luxuosas ilustradas. Há outras obras que apenas os especialistas conhecem, não só geógrafos como cultores de muitas outras ciências. Mas ele não foi apenas o escritor sugerido pela fotografia que escolheu como "retrato oficial" (fig. 1). Toda a vida, Orlando Ribeiro lutou para poder realizar investigações e para que o Centro de Estudos Geográficos, que criou em 1943 em Lisboa, sobrevivesse e se desenvolvesse. Lutou também para que a Universidade portuguesa atingisse e mantivesse um alto grau de excelência.

Entre a abundante documentação que se acumulou em casa e a que descansa, desigualmente arrumada nos arquivos ainda pouco explorados do Centro de Estudos Geográficos de Lisboa, escolheram-se cinco textos, de índole e destinatários diversos, datando de 1939, 1946, 1968, 1974 e 1978, por se afigurarem capazes de sugerir, aos leitores mais novos, um pouco do que foi a trajectória e a personalidade combativa, entusiasta e, tantas vezes, amargurada, do criador do Centro de Estudos Geográficos.

O primeiro documento escolhido, ao contrário dos seguintes, não é um inédito, por ter sido divulgado há já dois terços de século, na Revista da Faculdade de Letras, n. ${ }^{\circ}$ VII 1-2, em 1941. Foi escrito durante as férias da Páscoa de 1939, ou seja, durante o segundo ano da estadia de Orlando Ribeiro em Paris, como bolseiro do Instituto para a Alta Cultura (IAC) e leitor de português na Sorbonne. Ele estava então a completar, no Institut de Géographie de la rue Saint-Jacques, a sua formação de geógrafo (Ribeiro, 2003: 80-89; Daveau, 2007 a), e a pensar já no seu futuro papel de animador da investigação geográfica em Portugal.

A nota reproduzida é, antes de tudo, uma clara síntese das variadas formas que assumiam então os Deslocamentos de população em Portugal. Este tema de

* Recebido: 10/12/2007. Revisto: 23/01/2008. Aceite: 27/02/2008.

1 Os textos de Orlando Ribeiro foram coligidos e são aqui apresentados por Suzanne Daveau, Investigadora do Centro de Estudos Geográficos da Universidade de Lisboa. E-mail: sdaveau@clix.pt 
estudo tinha sido proposto, em Julho de 1938, no Congresso Internacional de Geografia de Amesterdão, no qual tinha participado o jovem geógrafo. Lembrando os seus inspiradores metodológicos (Faucher e Siegfried, em França, Leite de Vasconcellos e Viera da Silva, em Portugal), ele apoiou-se também nas primeiras observações próprias, realizadas durante viagens de estudo através de Portugal, sistematicamente encetadas no Verão de 1936. Aparece no texto um apanhado bem organizado dos vários aspectos do tema, tal como era visto na altura, mas não ainda uma proposta concreta de investigação. Com efeito, é interessante notar que o autor tinha, então, plena consciência do muito que faltava para "o futuro trabalho de síntese, que por ora seria prematuro tentar". Tratava-se tão só de "multiplicar exemplos e preparar o material", utilizando para isso, na falta de colegas ou discípulos então inexistentes, a boa vontade de "estudiosos ou simples curiosos". Já no ano anterior, 1938, Orlando Ribeiro tinha conseguido difundir largamente, através do IAC, dois Inquéritos impressos sobre o "Habitat rural" e a "Geografia regional", duas tentativas para sistematizar e oficializar, ao mesmo tempo, a técnica de recolha de dados por inquéritos, que tinha aprendido, a partir de 1931, na companhia estudiosa de Leite de Vasconcellos.

O segundo documento é um memorial, escrito em 1946, mas que ficou então sem título. Em 1969 o autor chamar-lhe-ia Propósitos e projectos da minha carreira de geógrafo. Ainda que cuidadosamente composto e passado à máquina, não há certeza de ele ter sido mandado, em 1946, a "suas Ex ${ }^{\text {as }}$ os Ministros das Colónias e da Educação Nacional", aos quais estava dirigido. Quando o reviu e anotou cuidadosamente, em 1969, Orlando Ribeiro considerou-o como um "Relatório inédito", a incluir na colectânea que tinha então em preparação. Por sugestão do editor e amigo João Sá da Costa, ele estava começando a preparação de três volumes de Ensaios de Geografia Humana e Regional, onde acolheria as suas já numerosas obras inéditas ou avulsas. Este texto estava muito provavelmente destinado a figurar no Volume III, dentro da rubrica tratando do "ofício de geógrafo, no duplo aspecto da reflexão e da acção" (p. 2 da capa do Volume I). Na realidade, apenas se publicará, em 1970, o Volume I da colectânea projectada. O presente memorial, possivelmente por ter ficado entretanto "sedimentado" entre outras numerosas papeladas, não figura também nos seis volumes de Opúsculos Geográficos, que a Fundação Gulbenkian editaria, de 1989 a 1995.

Para manter inteligível a dualidade temporal da preparação deste texto, utilizaram-se as seguintes convenções: as palavras, grupos de palavras ou páginas escritas em 1946, mas riscadas em 1969, são mantidas aqui entre parênteses rectos; os acrescentos ou correcções de 1969 aparecem em itálico, exceptuando-se apenas as pequenas emendas de gralhas; as palavras sublinhadas no texto original continuam com a mesma grafia no presente texto.

Não se encontrou infelizmente, até agora, o "relatório seguinte", que dataria de 1955 e ao qual o autor faz alusão na sua nota 5, escrita em 1969. Mas o presente texto afigura-se já muito esclarecedor dos começos do Centro 


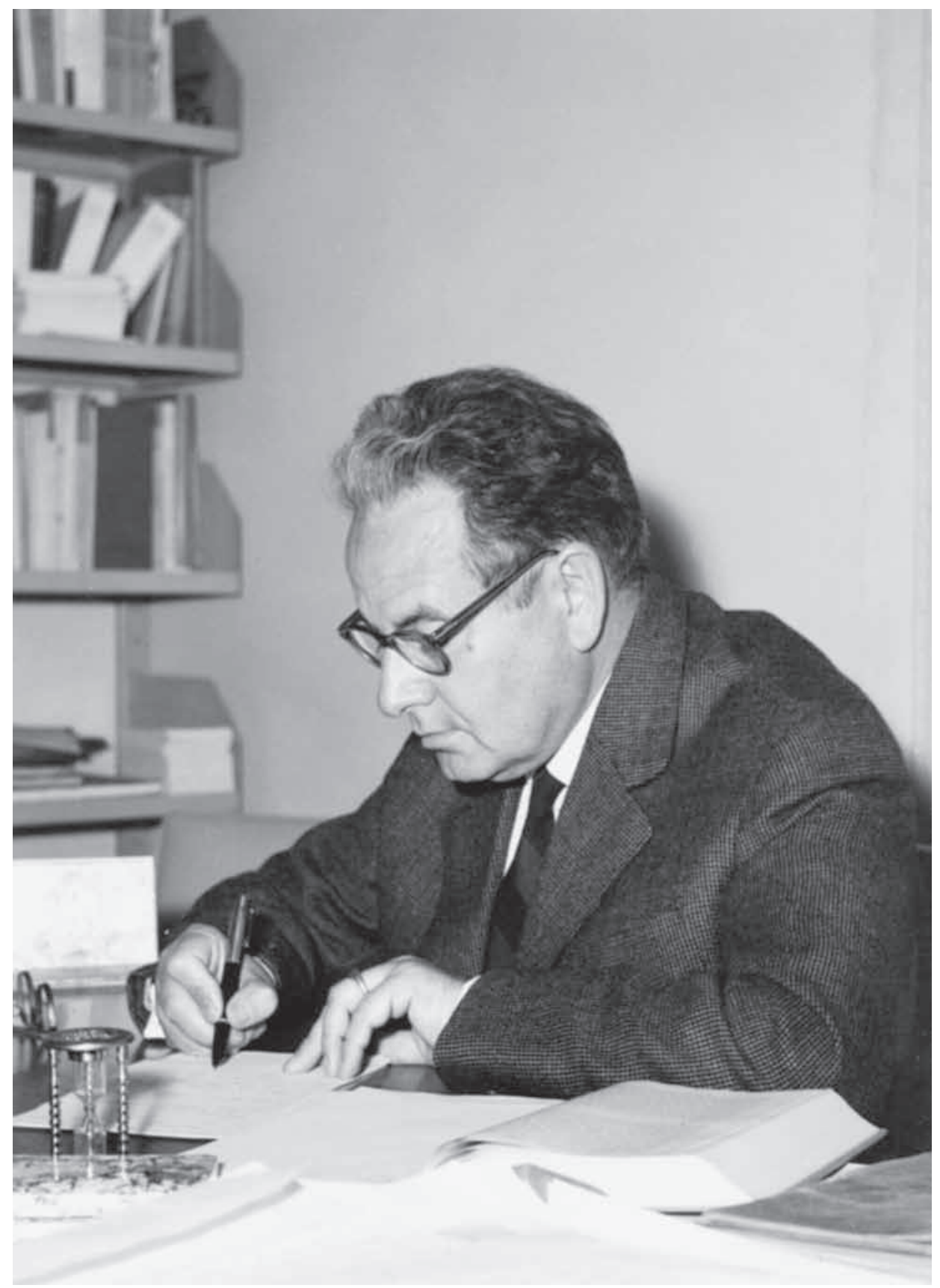

Fig. 1 - Retrato, dito "oficial”, de Orlando Ribeiro escrevendo, em 1965.

(Foto: José Pereira)

Fig. 1 - Portrait of Orlando Ribeiro in 1965. 
de Estudos Geográficos, criado formalmente em 1943, mas que apenas começou a receber subsídios em 1945. No ano seguinte, Orlando Ribeiro não tinha ainda conseguido formar discípulos, mas acabava de ter a grande alegria de encontrar um valioso colaborador, o Eng. ${ }^{\circ}$ Mariano Feio. Para aligeirar o peso da própria responsabilidade científica, teve então a tentação de dividir a Geografia em duas áreas: a parte física a cargo de Mariano Feio e a parte humana a seu cargo. Mas acabou por não ceder à tentação como apontou ironicamente em 1969, na sua nota 2. Entre 1947 e 1950, todas as forças do jovem Centro de Investigações serão dirigidas à preparação do Congresso Internacional de Geografia de 1949 e ao resultante rescaldo editorial, o que explica, em boa parte, a renúncia ou o protelamento de parte dos projectos enunciados em 1946 (Daveau, 2007 b).

O terceiro documento reproduzido é uma Nota aos Colaboradores Científicos do Centro de Estudos Geográficos. Em 30 de Setembro de 1968 ela foi distribuída, policopiada, aos 14 investigadores que contava então o Centro. Além do próprio Director, do Prof. Ilídio do Amaral, de mim e do secretário do Centro, Dr. Machado Guerreiro, trabalhavam já ali dez licenciados: Fernando Rebelo (de Coimbra), António de Brum Ferreira, Proença Ribeiro, Carminda Cavaco, Isabel Medeiros, Carlos Alberto Medeiros, Jorge Gaspar, Maria Alfreda Cruz, Paula Lema e Galopim de Carvalho (fig. 2). Esta Nota acompanhava a retomada actividade de Director do Centro, a seguir ao ano escolar passado em Paris como Professor convidado na Sorbonne e ao Verão de convalescença depois de gravosa operação. Neste texto, transparece não apenas a amizade e o à-vontade das relações entre um Professor, já então com 57 anos de idade, e os colaboradores mais jovens, como a extrema exigência científica que lhes desejava incutir. Não para lhes tirar a "autonomia científica", que respeitou sempre escrupulosamente, mas para manter no mais alto nível científico a investigação praticada e para não deixar aumentar os aspectos perigosos da crescente especialização científica. Porque, não desejando "impedir a especialização de cada um (...) tenho o dever de manter a unidade de atitude e a coesão científica do nosso agrupamento."

O quarto documento foi distribuído, como ainda bem me lembro, no meio de uma das inúmeras reuniões agitadas, que se sucediam tanto ao nível da Faculdade como do Centro, nas semanas posteriores à Revolução de 25 de Abril de 1974. Os mais novos, entusiasmados e cheios de inocência e de ignorância social, facilmente explicáveis por quarenta anos de ditadura e de mutismo, acreditavam que iam criar, de repente, um mundo novo e bem mais justo. Tomavam múltiplas decisões por aclamações, num ambiente de grande fervor e de total improvisação. Muitos professores estavam ausentes, outros partilhavam do entusiasmo dos mais novos e outros, ainda, acediam a tudo o que era proposto, por comodismo ou cobardia.

Orlando Ribeiro nunca foi cobarde e, na impossibilidade de se fazer ouvir, redigiu e mandou difundir na sala de reuniões, no dia 17 de Maio, um pequeno texto tratando de A Situação Actual da Faculdade. Esta iniciativa foi bastante mal 


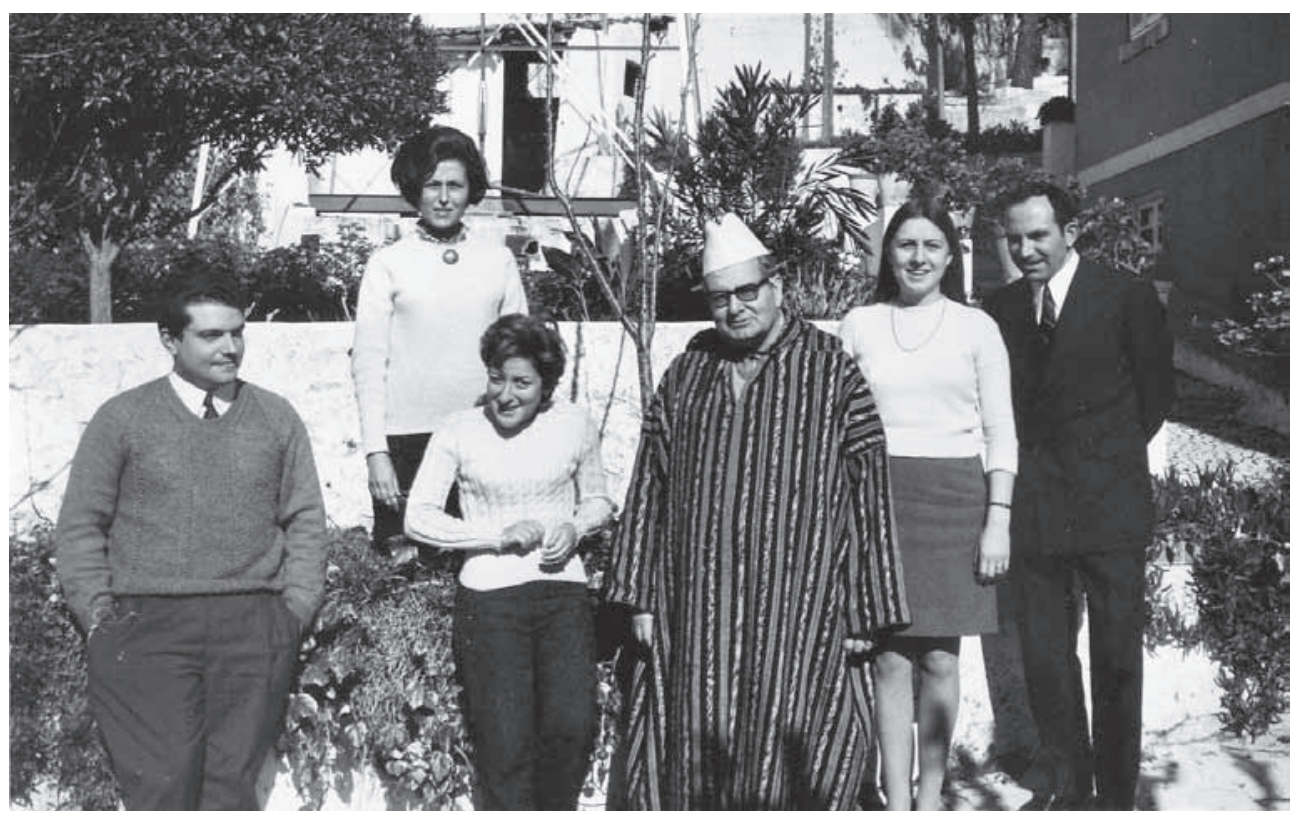

Fig. 2 - Orlando Ribeiro, vestido de mouro, recebe no quintal de Vale de Lobos alguns jovens colaboradores, no começo dos anos 70. Da esquerda para a direita, Carlos Alberto Medeiros, Carminda Cavaco, Isabel Medeiros, Denise e António de Brum Ferreira.

Fig. 2 - Orlando Ribeiro, dressed like an arab, with young colleagues, in the 1970s in his Vale de Lobos house.

recebida: como era possível que um dos ultrapassados "catedráticos" se permitisse, sem autorização prévia do plenário, utilizar a máquina de policopiar para difundir as próprias ideias? Se ele pode ainda notar com agrado que: "Não foi até agora pedido o afastamento de nenhum docente da secção de Geografia", bem pouco tempo ia decorrer até que - porventura em razão mesmo da difusão desta nota -, lhe fosse, como a tantos outros colegas, instaurado um processo de saneamento, que lhe será notificado apenas um ano mais tarde, em 17 de Junho de 1975. Chamar os alunos e colegas "ao trabalho aturado e à reflexão profunda", recusar tanto o "deplorável nivelamento pela mediocridade no estudo" como "a paralisação das aulas", eram declarações que não podiam ser bem recebidas naquela altura.

Em anexo à nota policopiada difundida no dia 17 de Maio, encontrei uma folhinha manuscrita, provavelmente escrita no dia seguinte, fundamentando para ele próprio a decisão então tomada: "As propostas dos alunos, abaixando a classificação da dispensa de exame, suprimindo desde já qualquer ensino e privando portanto alguns alunos do seu direito de aprender, eliminando a dissertação de licenciatura (que efectivamente se devia aligeirar, apressar e simplificar), representam um propósito de interromper os trabalhos escolares. Como professor e como investigador encontro-me assim impossibilitado de cumprir o meu dever e a minha vocação; não vejo qualquer base possível de discussão se os 
professores cederem à pressão no seu objectivo fundamental e primacial de ensinar; não desejo, como homem livre que procura tomar clara consciência dos seus direitos e obrigações, ceder a formas de pressão tendenciosas e não esclarecidas. Nesta conformidade, não vou tirar mais tempo a trabalhos pessoais, entre os quais à redacção das minhas próprias lições de Geografia humana que os alunos me impediram de continuar".

O quinto documento foi dirigido, em 19 de Junho de 1978, Ao Conselho Cientifico do Centro de Estudos Geográficos, ou seja, aos investigadores já doutorados. Refere-se a uma das numerosas fases em que a actividade científica do Centro se encontrou seriamente ameaçada por decisões arbitrárias das entidades administrativas, de que dependeu sucessivamente. Desta vez, tratava-se de uma tentativa de reorganização do Instituto Nacional de Investigação Científica (INIC), sucessor do Instituto de Alta Cultura (IAC), no quadro do qual o Centro de Estudos Geográficos tinha sido criado em 1943. Num desejo de uniformização, o INIC entendeu de repente, em 1978, incorporar as publicações do Centro num sistema geral de edição, evidentemente não preparado para as gerir. Por sorte, acabava então de ser nomeado Secretário de Estado da Investigação Científica o Professor Sousa Pinto, que quis ouvir a opinião do Director do Centro. No discurso proferido no dia do seu jubileu, em 16 de Fevereiro de 1981, Orlando Ribeiro contará como este "investigador distinto da fisiologia da audição, teve comigo uma conversa de hora e meia que me deixou as maiores esperanças, se os alcatruzes da política o não tivessem pouco tempo depois metido debaixo de água." (Memórias..., 2003: 159).

Deste episódio - um entre tantos outros, que impediram o Centro de Estudos Geográficos de obter continuidade e segurança suficientes na gestão dos seus empreendimentos científicos - sobrevivem duas notas, uma através da qual Orlando Ribeiro deu conta aos seus colaboradores, em 19 de Junho 1978, do encontro havido e outra, datada de 12 de Junho. Este segundo texto tem o duplo interesse de fazer o ponto da situação sobre a produção científica do Centro em 1978 e de evocar o áureo período durante o qual ele beneficiou de largos apoios da Fundação Gulbenkian (fig. 3). Período que chegava então ao fim - como Orlando Ribeiro visivelmente já adivinhava, embora sem aceitar ainda plenamente a nova "situação catastrófica" e continuando a gabar o "auxílio material precioso que a Fundação nos tem prestado e parece disposta a continuar".

\section{OBRAS CITADAS}

Daveau S (2007 a) La Géographie, ça peut survivre à la guerre. Finisterra - Revista Portuguesa de Geografia, XLII(83): 5-20.

Daveau S (2007 b) Planeamento e difusão das obras de Orlando Ribeiro In Geophilia - o sentir e os sentidos da Geografia, CEG, Lisboa: 171-183.

Ribeiro O (2003) Memórias de um Geógrafo. Edições João Sá da Costa, Lisboa. 


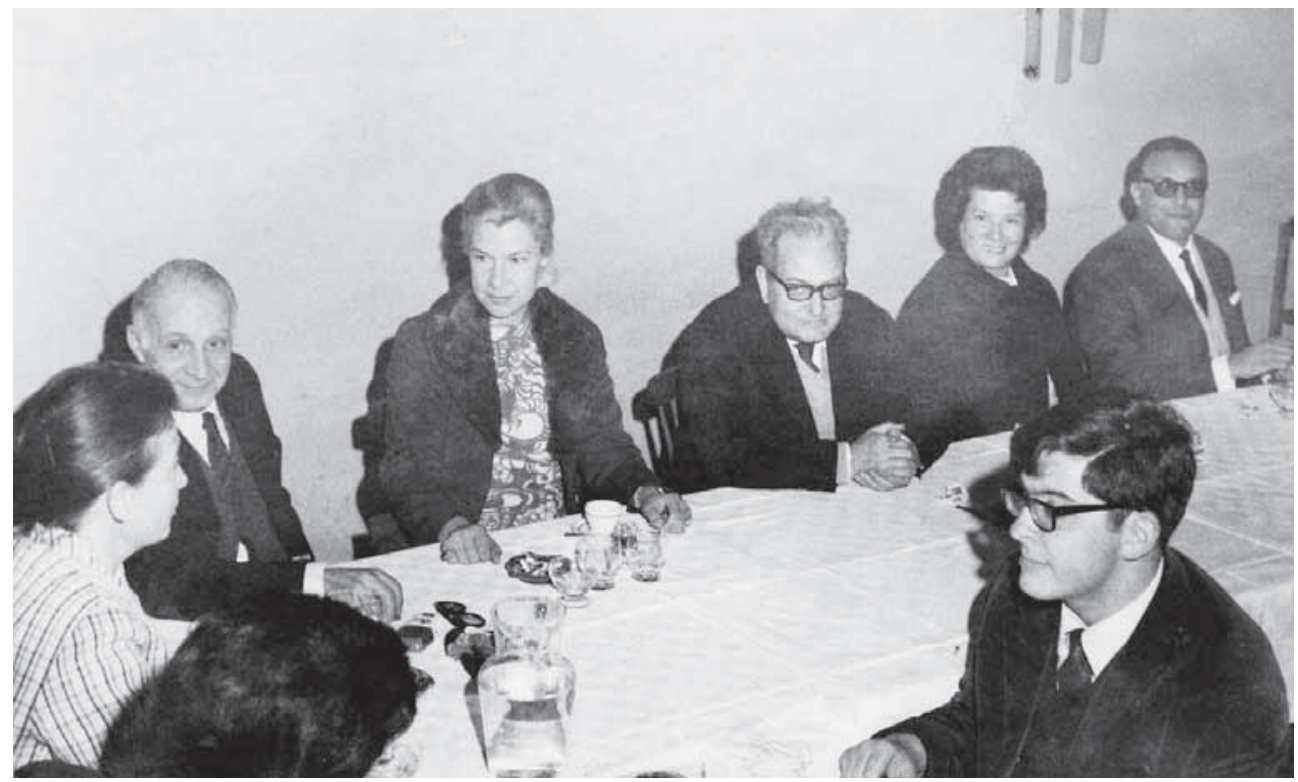

Fig. 3 - A mesa de honra, num almoço na Quinta de S. Vicente, em 11 de Fevereiro de 1970. Da esquerda para a direita, Suzanne Daveau, José Ribeiro dos Santos, administrador da Fundação Gulbenkian, Raquel Soeiro de Brito, Orlando Ribeiro, Margarida Ribeiro e Ilídio do Amaral; em primeiro plano, o filho Manuel.

Fig. 3 - Formal lunch of the CEG staff in 1970.

\section{LE CHERCHEUR, LE PROFESSEUR UNIVERSITAIRE ET LE DIRECTEUR DU CENTRE D'ÉTUDES GÉOGRAPHIQUES}

Orlando Ribeiro est surtout connu en tant qu'auteur de textes géographiques sur le Portugal et les pays lusophones. Certains de ces textes connurent une large diffusion, sous forme d'extraits dans des manuels scolaires ou dans ces albums illustrés qu'on offre en fin d'année. D'autres ne sont connus que par les spécialistes de rameaux scientifiques divers. Mais il ne fut pas qu'un excellent auteur, alliant la rigueur scientifique à la beauté de l'expression. L'impulsion qu'il donna à la recherche universitaire, surtout dans le cadre du Centre d'Études Géographiques créé par lui en 1943, est un aspect au moins aussi important de sa vie professionnelle.

Sont présentés ici cinq textes anciens, retrouvés dans ses archives personnelles ou dans celles du Centre. Ils permettent de faire revivre ce que furent ses persistants efforts pour accroître la qualité de l'enseignement et de la recherche universitaires au Portugal.

Le premier texte n'est pas inédit, mais il a été choisi pour être le projet de recherches le plus anciennement diffusé par Orlando Ribeiro, alors qu'il complétait encore sa formation à l'Institut de Géographie de la rue Saint Jacques, à Paris. C'est une excellente synthèse sur la façon dont était alors envisagée l'étude des mouvements de population, thème d'étude déjà amorcé par lui au Portugal sous l'influence de Leite de Vasconcellos et qui venait d'être proposé internationalement lors du Congrès de Géographie d'Amsterdam, en 1938. Il s'agissait seulement de réunir des informations, en utilisant des 
questionnaires bien préparés, comme ceux qu'il venait de diffuser sur l'Habitat rural et sur la Géographie régionale au Portugal. La synthèse serait pour plus tard.

Le second texte est un mémoire destiné aux autorités ministérielles de tutelle et de financement du Centre d'Études Géographiques. Écrit en 1946, il fut retouché en 1969 pour une éventuelle publication. Intitulé alors Espoirs et projets de ma carrière de géographe, il donne une bonne idée de ce que furent les débuts du Centre d'Études Géographiques de Lisbonne. L'absence presque complète d'étudiants en Géographie rendait difficile le recrutement de jeunes collaborateurs. Celui, inespéré, de Mariano Feio, ingénieur et paléontologue, fut donc une chance pour le Centre. Elle incita alors Orlando Ribeiro à envisager une partition de la direction scientifique, entre Géographie physique et humaine. En fait, cette division malheureuse ne s'amorcera que bien plus tard. Tous les efforts étaient alors tournés vers la préparation du Congrès International de Géographie de 1949.

Le troisième texte est une petite note, adressée en septembre 1968 aux 14 géographes et autres chercheurs que rassemblait alors le Centre. Orlando Ribeiro rappelait amicalement mais fermement, à ses jeunes collègues, l'obligation qu'ils avaient d'atteindre un haut niveau scientifique et aussi de collaborer aux tâches d'intérêt collectif. La spécialisation de chacun dans sa propre recherche ne devait pas empêcher «de maintenir l'unité de point de vue et la cohésion scientifique de notre groupe.»

Le quatrième texte fut distribué, le 17 mai 1974, au cours d'une des nombreuses réunions agitées d'étudiants et de professeurs qui suivirent la révolution du 25 avril. Orlando Ribeiro y rappelait qu'il était impératif de reprendre rapidement un rythme de travail soutenu: enseigner, étudier, passer les examens et approfondir la réflexion avant de prendre des décisions révolutionnaires. Ce point de vue fut, bien sûr, fort mal reçu et c'est peut-être ce qui explique qu'il ait été, quelques jours plus tard, l'objet d'une dénonciation tendant à un procès d'épuration. Il notait alors, un peu amèrement, qu'en l'absence d'élèves, il allait pouvoir se consacrer tout entier à ses recherches et à la mise au net de ses cours.

Le cinquième texte illustre une des phases administrativement difficiles, auxquelles le Directeur du Centre d'Études Géographiques eut à faire face de façon récurrente. En 1978, l'organisme de tutelle des groupes de recherche au Portugal, alors désigné par le sigle INIC, entendait centraliser et uniformiser les publications des divers centres, sans avoir pris en compte l'importance et la spécificité des publications du CEG. Celui-ci jouissait alors d'une phase prospère, encore que précaire, due aux subventions accordées par la Fondation Gulbenkian. Les deux notes conservées à ce sujet font ainsi renaître un des moments de la lutte incessante qui permit à Orlando Ribeiro de maintenir en vie et de voir prospérer le Centre d’Études Géographiques de Lisbonne.

\section{THE RESEARCHER, THE UNIVERSITY TEACHER AND THE DIRECTOR OF THE CENTRO DE ESTUDOS GEOGRÁFICOS}

Orlando Ribeiro is known chiefly as the author of geographical writings about Portugal and other Portuguese-speaking countries. Some of these writings have been included in handbooks and deluxe illustrated albums. Scholars from a variety of scientific disciplines have relied abundantly on the rest. However, he was not only a good 
writer, at once scientific and pleasant. The impulse that he gave to research, chiefly through the research centre that he created in 1943, CEG, is another very important side of his professional life.

Five papers, collected from his personal and professional records, are presented here. They bring back to life his efforts to improve the level of teaching and research in the Portuguese universities.

The first paper was chosen because it is the first research outline prepared by Orlando Ribeiro, while he was still undergoing training at the Paris Geographical Institute. It synthesizes the ideas prevalent at the time on the subject of human migration, as based on Leite de Vasconcellos' lectures in Portugal and the treatment of the topic as of the International Geographical Congress in Amsterdam (1938). Data collection was expected to rely on survey methodologies such as those carried out by Orlando Ribeiro on the topics of Rural Settlement and Regional Geography in Portugal.

The second paper was addressed to the governmental offices that managed and funded the C.E.G. It was written in 1946 and revised for publishing in 1969. Entitled "Hopes and aims of my life as a geographer", it provides a vivid picture of the early days of C.E.G. At that time, there were both very few students and very few researchers. The admission of Mariano Feio, a fully-trained engineer and palaeontologist, provided a fortunate opportunity. Orlando Ribeiro decided at the time to share the scientific management of the C.E.G. with Mariano Feio, who would head the Physical Geography branch. Orlando Ribeiro himself would carry on heading the Human Geography one. This disastrous division would not enter into practice, however, due to the fact that the small research group was fully focused on preparing the 1949 International Geographical Congress.

The third paper was addressed to the 14 research fellows who worked at the C.E.G. in 1968. Orlando Ribeiro corteously reminded them that the increasingly specialized character of their own research should not prove an obstacle to the necessary collaboration and teamwork, nor to the mandatory constant quest for personal scientific improvement. Upholding the scientific cohesion of the research group was the overarching goal.

The fourth paper was distributed during one of the lively meetings that took place shortly after the Revolution of April $25^{\text {th }}$, 1974. In it, Orlando Ribeiro sought to explain to his restless students and colleagues that, indeed, they had to work hard, teach and study, examine and sit in exams, and that a true revolution had to rest on thorough considerations. Naturally, this opinion did not prove popular at all. In fact, it may have provided the grounds for the accusation of counter-revolutionary activities that was internally laid against him a few days later. Orlando Ribeiro decided that, should it prove impossible to carry on teaching, he would be content with laying down in writing his lectures and investigations.

The final documents in this collection refer to a flourishing but diffimer moment in the life of the C.E.G., which took place in 1978. INIC, the National Office for Scientific Research, then decided, rather unexpectedly, to unify the publishing rules and procedures of all the research centres that depended on its funding. In so doing, it ignored the specificity and importance of C.E.G.'s long-lasting publishing tradition. Orlando Ribeiro had to fight hard to uphold the autonomy and personality of his research centre, at the very time when the funding from the Gulbenkian Foundation was unfortunately and seriously at risk. 